\title{
Non-Classical Formulation of Photon Energy for the Degenerate Parametric Oscillator
}

\author{
Solomon G. Belete \\ Physics Department, Jimma University, Jimma, Ethiopia \\ Email: solgett@yahoo.com, solgett@gmail.com
}

Received 18 June 2014; revised 15 July 2014; accepted 9 August 2014

Copyright (C) 2014 by author and Scientific Research Publishing Inc.

This work is licensed under the Creative Commons Attribution International License (CC BY). http://creativecommons.org/licenses/by/4.0/

c) (i) Open Access

\begin{abstract}
We have analyzed photon statistics and quadrature squeezing of the signal mode produced by one-mode subharmonic generator. It is found that the mean photon energy of the signal mode is twice of a twin signal light beam. And the energy fluctuations of the two signal light beams are four times that of one of the signal light beams. We have also shown that the photon energy quadrature squeezing of the output signal mode is exactly equal to that of the cavity signal mode. In light of this, the global quadrature squeezing of the signal mode is independent of the mean photon energy. On the basis of the above results, the quantum analysis of a one-mode sub-harmonic generating system should somehow be modified.
\end{abstract}

\section{Keywords}

Signal Mode, A Twin Signal Light Beam, Photon Energy, Energy Fluctuations, Energy Quadrature Squeezing

\section{Introduction}

A one-mode sub-harmonic generator is one of the most interesting and well-studied quantum optical systems. This system consists of a nonlinear crystal which is pumped by coherent light and placed inside a cavity coupled to a vacuum reservoir. This quantum optical process leads to the generation of squeezed light. A theoretical analysis of the quadrature squeezing and photon statistics of a signal mode produced by a one-mode sub-harmonic generator has been made by a number of authors [1]-[9]. It has been established that the signal mode has a maximum of $50 \%$ squeezing below the coherent-state level [1]-[4].

In a one-mode sub-harmonic generator, a pump photon of frequency $2 \omega$ is down converted into a pair of signal photons each of frequency $(\omega)$. It is to be recalled that the Hamiltonian describing the process of sub-harmonic generation consists of the operators $\hat{a}^{2}$ and $\hat{a}^{+2}$. And the quantum analysis of the signal mode is 
usually carried out employing the operator's $\hat{a}$ and $\hat{a}^{+}$. However, such analysis leads, among others, to onehalf of the mean photon number of the signal mode [2] [4]-[8] [10]-[14]. This is surely the mean number of one set of the signal photons (a twin signal light beam), consisting of one photon from each pair [1]. Since the other set of the signal photons is not included in such analysis, we seek to resolve this problem by superposing the $Q$ function of the two set of signal photons (the signal mode), each set consisting of one photon from each pair. The resulting $Q$ function is used to determine the mean photon number, the mean energy, the variance of the photon number, the energy fluctuations, the photon number quadrature variance, the photon energy quadrature fluctuations, the photon number quadrature squeezing and the photon energy quadrature squeezing of the two signal light beams.

\section{The Q Function}

We seek first to determine the $Q$ function for the signal mode produced by one-mode sub-harmonic generator. This can be done by superposing the $Q$ functions of the two sets of signal photons, each set consisting of one signal photon from each pair.

We recall that the $Q$ function for the signal light beam has the form [1]

$$
Q\left(\alpha^{*}, \alpha, t\right)=\frac{\left[l^{2}-m^{2}\right]^{1 / 2}}{\pi} \exp \left[-l \alpha^{*} \alpha-\frac{m}{2}\left(\alpha^{* 2}+\alpha^{2}\right)\right],
$$

where

$$
\begin{aligned}
& l=\frac{c}{c^{2}-d^{2}}, \\
& m=\frac{d}{c^{2}-d^{2}},
\end{aligned}
$$

with

$$
\begin{gathered}
c=1-\frac{\varepsilon}{2(\kappa+2 \varepsilon)}\left(1-\mathrm{e}^{-(\kappa+2 \varepsilon) t}\right)+\frac{\varepsilon}{2(\kappa-2 \varepsilon)}\left(1-\mathrm{e}^{-(\kappa-2 \varepsilon) t}\right), \\
d=\frac{\varepsilon}{2(\kappa+2 \varepsilon)}\left(1-\mathrm{e}^{-(\kappa+2 \varepsilon) t}\right)+\frac{\varepsilon}{2(\kappa-2 \varepsilon)}\left(1-\mathrm{e}^{-(\kappa-2 \varepsilon) t}\right) .
\end{gathered}
$$

Here, $\varepsilon$ and $\kappa$ are the amplitude of the driving coherent light and the cavity damping constant, respectively.

We now proceed to determine the $Q$ function for the two signal light beams produced by one-mode sub-harmonic generators. The $Q$ function for the superposition of two light beams can be defined by [1]

$$
Q\left(\alpha^{*}, \alpha, t\right)=\frac{1}{\pi}\left(\left\langle\alpha\left|\hat{\rho}_{2}\right| \alpha\right\rangle\right),
$$

is the c-number corresponding to normally ordered density operator divided by $\pi$. Suppose $\hat{\rho}\left(\hat{a}^{+}, \hat{a}\right)$ is density operator for a certain light beam. Then upon expanding the density operator in normal order

$$
\hat{\rho}\left(\hat{a}^{+}, \hat{a}\right)=\frac{1}{\pi} \sum_{k l} C_{k l} \hat{a}^{+k} \hat{a}^{l} .
$$

And employing the completeness relation for coherent state

$$
\int \mathrm{d}^{2} \beta|\beta\rangle\langle\beta|=1,
$$

one can easily find

$$
\hat{\rho}\left(\hat{a}^{+}, \hat{a}\right)=\frac{1}{\pi} \int \mathrm{d}^{2} \beta \sum_{k l} C_{k l} \beta^{* k}|\beta\rangle\langle\beta| \hat{a}^{l}
$$


and using the identity

$$
|\beta\rangle\left\langle\beta\left|\hat{a}^{l}=\left(\beta+\frac{\partial}{\partial \beta^{*}}\right)^{l}\right| \beta\right\rangle\langle\beta|,
$$

there follows

$$
\hat{\rho}\left(\hat{a}^{+}, \hat{a}\right)=\frac{1}{\pi} \int \mathrm{d}^{2} \beta \sum_{k l} C_{k l} \beta^{* k}\left(\beta+\frac{\partial}{\partial \beta^{*}}\right)^{l}|\beta\rangle\langle\beta| .
$$

This expression can be put in terms of the displacement operator in the form

$$
\hat{\rho}\left(\hat{a}^{+}, \hat{a}\right)=\frac{1}{\pi} \int \mathrm{d}^{2} \beta \sum_{k l} C_{k l} \beta^{* k}\left(\beta+\frac{\partial}{\partial \beta^{*}}\right)^{l} \hat{D}(\beta)|0\rangle\langle 0| \hat{D}(-\beta) .
$$

We now realize that the density operator for the superposition of the first light beam and another one expressible as [1]

$$
\hat{\rho}_{2}=\frac{1}{\pi} \int \mathrm{d}^{2} \gamma \sum_{m n} C_{m n} \gamma^{* m}\left(\gamma+\frac{\partial}{\partial \gamma^{*}}\right)^{n} \hat{D}(\gamma) \hat{\rho}\left(\hat{a}^{+}, \hat{a}\right) \hat{D}(-\gamma),
$$

so that in view of (12), we have

$$
\hat{\rho}_{2}=\frac{1}{\pi^{2}} \int \mathrm{d}^{2} \beta \mathrm{d}^{2} \gamma Q_{1}\left(\beta^{*}, \beta+\frac{\partial}{\partial \beta^{*}}, t\right) Q_{2}\left(\gamma^{*}, \gamma+\frac{\partial}{\partial \gamma^{*}}, t\right)|\beta+\gamma\rangle\langle\gamma+\beta|,
$$

in which

$$
Q_{1}\left(\beta^{*}, \beta+\frac{\partial}{\partial \beta^{*}}, t\right)=\frac{1}{\pi} \sum_{k l} C_{k l} \beta^{* k}\left(\beta+\frac{\partial}{\partial \beta^{*}}\right)^{l}
$$

and

$$
Q_{2}\left(\gamma^{*}, \gamma+\frac{\partial}{\partial \gamma^{*}}, t\right)=\frac{1}{\pi} \sum_{m n} C_{m n} \gamma^{*_{m}}\left(\gamma+\frac{\partial}{\partial \gamma^{*}}\right)^{n} .
$$

Now the combination of (6) and (14) give rise the $Q$ function for superposition of two light beams as in the form

$$
\begin{aligned}
& Q\left(\alpha, \alpha^{*}, t\right)=\frac{1}{\pi} \int \mathrm{d}^{2} \beta \mathrm{d}^{2} \gamma Q_{1}\left(\beta^{*}, \beta+\frac{\partial}{\partial \beta^{*}}, t\right) \mathrm{e}^{(\alpha-\beta-\gamma) \beta^{*}} Q_{2}\left(\gamma^{*}, \gamma+\frac{\partial}{\partial \gamma^{*}}, t\right) \\
& \times \mathrm{e}^{(\alpha-\beta-\gamma) \gamma^{*}} \mathrm{e}^{-\alpha^{*} \alpha+\alpha^{*} \beta+\alpha^{*} \gamma}
\end{aligned}
$$

Finally, applying the binomial theorem, one can readily establish the $Q$ function for a pair of superposed cavity light beams as [1]

$$
\begin{aligned}
& Q\left(\alpha, \alpha^{*}, t\right)=\frac{1}{\pi} \int \mathrm{d}^{2} \beta \mathrm{d}^{2} \gamma Q_{1}\left(\beta^{*}, \alpha-\gamma, t\right) Q_{2}\left(\gamma^{*}, \alpha-\beta, t\right) \\
& \times \exp \left[-\alpha^{*} \alpha-\beta^{*} \beta-\gamma^{*} \gamma+\alpha^{*} \beta+\alpha^{*} \gamma+\alpha \beta^{*}+\alpha \gamma^{*}-\beta^{*} \gamma-\gamma^{*} \beta\right],
\end{aligned}
$$

where

$$
Q_{1}\left(\beta^{*}, \beta+\frac{\partial}{\partial \beta^{*}}, t\right) \mathrm{e}^{(\alpha-\beta-\gamma) \beta^{*}}=Q_{1}\left(\beta^{*}, \alpha-\gamma, t\right) \mathrm{e}^{(\alpha-\beta-\gamma) \beta^{*}}
$$

and 


$$
Q_{2}\left(\gamma^{*}, \gamma+\frac{\partial}{\partial \gamma^{*}}, t\right) \mathrm{e}^{(\alpha-\beta-\gamma) \gamma^{*}}=Q_{2}\left(\gamma^{*}, \alpha-\beta, t\right) \mathrm{e}^{(\alpha-\beta-\gamma) \gamma^{*}}
$$

With the aid of Equation (1), the $Q$ function for one of the signal light beam can be put in the form

$$
Q_{1}\left(\beta^{*}, \alpha-\gamma, t\right)=\frac{\left[l^{2}-m^{2}\right]^{1 / 2}}{\pi} \exp \left[-l \beta^{*} \alpha+l \beta^{*} \gamma+m \alpha \gamma-\frac{m}{2} \beta^{* 2}-\frac{m}{2} \gamma^{2}-\frac{m}{2} \alpha^{2}\right] .
$$

And the $Q$ function for the other signal light beam can be written as

$$
Q_{2}\left(\gamma^{*}, \alpha-\beta, t\right)=\frac{\left[l^{2}-m^{2}\right]^{1 / 2}}{\pi} \exp \left[-l \gamma^{*} \alpha+l \gamma^{*} \beta+m \alpha \beta-\frac{m}{2} \gamma^{* 2}-\frac{m}{2} \alpha^{2}-\frac{m}{2} \beta^{2}\right] \text {. }
$$

Now introducing (21) and (22) into (18), we have

$$
\begin{aligned}
Q\left(\alpha, \alpha^{*}, t\right)= & \frac{\left[l^{2}-m^{2}\right]}{\pi} \exp \left[-\alpha^{*} \alpha-m \alpha^{2}\right] \times \int \frac{\mathrm{d}^{2} \beta}{\pi} \exp \left[-\beta^{*} \beta-l \beta^{*} \alpha-\frac{m}{2} \beta^{* 2}+m \alpha \beta+\beta^{*} \alpha+\alpha^{*} \beta\right] \\
& \times \int \frac{\mathrm{d}^{2} \gamma}{\pi} \exp \left[-\gamma^{*} \gamma+l \beta^{*} \gamma+l \gamma^{*} \beta-l \gamma^{*} \alpha-\frac{m}{2} \gamma^{* 2}-\frac{m}{2} \gamma^{2}+m \alpha \gamma+\gamma^{*} \alpha+\alpha^{*} \gamma-\beta^{*} \gamma-\beta \gamma\right] .
\end{aligned}
$$

Thus upon carrying out the integration over $\beta$ and $\gamma$, applying the relation [1]

$$
\int \frac{\mathrm{d}^{2} z}{\pi} \exp \left[a z z^{*}+b z+c z^{*}+A z^{2}+B z^{* 2}\right]=\left[\frac{1}{a^{2}-4 A B}\right]^{1 / 2} \exp \left[\frac{a b c+A c^{2}+B b^{2}}{a^{2}-4 A B}\right],
$$

we readily obtain

$$
Q\left(\alpha^{*}, \alpha, t\right)=\frac{1}{\pi}\left[\frac{l^{2}-m^{2}}{l^{2}-m^{2}-4 l+4}\right]^{1 / 2} \exp \left[-u \alpha^{*} \alpha-\left(-v\left(\alpha^{* 2}+\alpha^{2}\right)\right)\right]
$$

where

$$
u=\frac{m^{2}-l^{2}+2 l}{l^{2}-m^{2}-4 l+4}
$$

and

$$
v=\frac{m}{l^{2}-m^{2}-4 l+4} .
$$

One can easily check that the $Q$ function for the two signal light beams given by Equation (25) is normalized to unity.

\section{Photon Statistics}

In this section, we seek to study the statistical properties of the two signal light beams produced by the onemode sub-harmonic generators.

\subsection{The Mean Energy}

Here, we wish to calculate the mean photon number and the mean photon energy of the two signal light beams. The mean photon number, for the cavity light, is defined by

$$
\bar{n}=\left\langle\hat{a}^{+} \hat{a}\right\rangle \text {. }
$$

Then the mean photon number can be written in terms of the $Q$ function as 


$$
\bar{n}=\frac{1}{\pi} \int \mathrm{d}^{2} \alpha \mathrm{d}^{2} \beta Q\left(\beta, \alpha^{*}, t\right) \exp \left(-\alpha^{*} \alpha-\beta^{*} \beta+\alpha \beta^{*}+\beta \alpha^{*}\right) \beta^{*} \alpha .
$$

On account of Equation (25), expression (29) can be put in the form

$$
\bar{n}=\left.\frac{1}{\pi^{2}}\left[\frac{l^{2}-m^{2}}{l^{2}-m^{2}-4 l+4}\right]^{1 / 2} \frac{\mathrm{d}}{\mathrm{d} a} \int \mathrm{d}^{2} \alpha \mathrm{d}^{2} \beta \exp \left[-\alpha^{*} \alpha-\beta^{*} \beta+\alpha \beta^{*}+\beta \alpha^{*}+a \alpha \beta^{*}-u \alpha^{*} \beta-v \beta^{2}-v \alpha^{* 2}\right]\right|_{a=0},
$$

so that upon carrying out the integration, employing the relation given by (24), and performing the differentiation with respect to a and applying the condition $a=0$, we readily get

$$
\bar{n}=\frac{u}{u^{2}-4 v^{2}}-1 \text {. }
$$

Now in view of (26) and (27) along with (2) and (3), we easily find

$$
\bar{n}=2(c-1) \text {, }
$$

so that on account of (4), we have

$$
\bar{n}=\frac{\varepsilon}{(\kappa-2 \varepsilon)}\left(1-\mathrm{e}^{-(\kappa-2 \varepsilon) t}\right)-\frac{\varepsilon}{(\kappa+2 \varepsilon)}\left(1-\mathrm{e}^{-(\kappa+2 \varepsilon) t}\right) .
$$

Hence at steady state, we see that

$$
\bar{n}=\frac{4 \varepsilon^{2}}{\kappa^{2}-4 \varepsilon^{2}} .
$$

This is the mean photon number of the two signal light beams which is twice of the mean photon number obtained in Ref. [2]-[8].

On the other hand, for normally ordered operators and a cavity mode coupled to a vacuum reservoir, the output and cavity operators can be related by [1]

$$
\hat{a}_{\text {out }}(t)=\sqrt{\kappa} \hat{a}(t) .
$$

Then combining (28) and (35), we have

$$
\bar{n}_{\text {out }}=\kappa \bar{n} .
$$

This indicates that the mean photon number of the output light is $\kappa$ times that of the cavity light.

Moreover, we note that the quantum Hamiltonian of a single-mode light has the form [1]

$$
\hat{H}=\hat{E}^{+} \hat{E},
$$

where

$$
\hat{E}^{+}(t)=\sqrt{\hbar \omega} \hat{a}^{+}
$$

and

$$
\hat{E}(t)=\sqrt{\hbar \omega} \hat{a},
$$

so that the mean photon energy of the two signal light beams can be put as

$$
\bar{E}(t)=\langle\hat{H}\rangle=\left\langle\hat{E}^{+}(t) \hat{E}(t)\right\rangle=\hbar \omega \bar{n},
$$

in which $\hat{E}^{+}(t)$ and $\hat{E}(t)$ are energy raising and lowering operators. Then combining (34) and (40), we have

$$
\bar{E}(t)=\frac{4 \hbar \omega \varepsilon^{2}}{\kappa^{2}-4 \varepsilon^{2}},
$$


where $\omega$ is the frequency of one of the signal light beam. This indicates that the mean energy is equal to the product of photon energy and the mean photon number.

\subsection{The Energy Fluctuations}

We next proceed to obtain the variance of the photon number and the energy fluctuations for the two signal light beams produced by the one-mode sub-harmonic generator. The variance of the photon number, for the cavity light, is defined by

$$
(\Delta n)^{2}=\left\langle\left(\hat{a}^{+} \hat{a}\right)^{2}\right\rangle-\bar{n}^{2} .
$$

Using the commutation relation

$$
\left[\hat{a}, \hat{a}^{+}\right]=2,
$$

which holds true for a pair of superposed light beams, we find

$$
(\Delta n)^{2}=\left\langle\hat{a}^{+2} \hat{a}^{2}\right\rangle+2 \bar{n}-\bar{n}^{2} .
$$

Thus with the aid of Equation (25), the expectation value of $\hat{a}^{+2} \hat{a}^{2}$ can be written as

$$
\begin{aligned}
& \left\langle\hat{a}^{+2} \hat{a}^{2}\right\rangle \\
& =\left.\frac{1}{\pi^{2}}\left[\frac{l^{2}-m^{2}}{l^{2}-m^{2}-4 l+4}\right]^{1 / 2} \frac{\mathrm{d}^{2}}{\mathrm{~d} a^{2}} \int \mathrm{d}^{2} \alpha \mathrm{d}^{2} \beta \exp \left[-\alpha^{*} \alpha-\beta^{*} \beta+\alpha \beta^{*}+\beta \alpha^{*}+a \alpha \beta^{*}-u \alpha^{*} \beta-v \beta^{2}-v \alpha^{* 2}\right]\right|_{a=0}
\end{aligned}
$$

so that upon carrying out the integration, employing the relation given by (24), and performing the differentiation with respect to a and applying the condition $a=0$, we readily obtain

$$
\left\langle\hat{a}^{+2} \hat{a}^{2}\right\rangle=\frac{8 l^{2}}{\left(l^{2}-m^{2}\right)^{2}}+\frac{4 m^{2}}{\left(l^{2}-m^{2}\right)^{2}}-\frac{16 l}{l^{2}-m^{2}}+8 .
$$

Therefore, in view of (2) and (3), there follows

$$
\left\langle\hat{a}^{+2} \hat{a}^{2}\right\rangle=8 c^{2}+4 d^{2}-16 c+8 .
$$

Now combination of (32), (44), and (47) yields

$$
(\Delta n)^{2}=4\left(c^{2+} d^{2}-c\right) .
$$

Then with the aid of Equations (4) and (5), we obtain

$$
\begin{aligned}
(\Delta n)^{2}= & \frac{2 \varepsilon^{2}}{(\kappa+2 \varepsilon)^{2}}\left[1-\mathrm{e}^{-(\kappa+2 \varepsilon) t}\right]^{2}+\frac{2 \varepsilon^{2}}{(\kappa-2 \varepsilon)^{2}}\left[1-\mathrm{e}^{-(\kappa-2 \varepsilon) t}\right]^{2} \\
& -\frac{2 \varepsilon}{(\kappa+2 \varepsilon)}\left(1-\mathrm{e}^{-(\kappa+2 \varepsilon) t}\right)+\frac{2 \varepsilon}{(\kappa-2 \varepsilon)}\left(1-\mathrm{e}^{-(\kappa-2 \varepsilon) t}\right),
\end{aligned}
$$

which at steady-state turns out to be

$$
(\Delta n)^{2}=\frac{16 \varepsilon^{4}}{\left(\kappa^{2}-4 \varepsilon^{2}\right)^{2}}+\frac{4 \kappa^{2} \varepsilon^{2}}{\left(\kappa^{2}-4 \varepsilon^{2}\right)^{2}}+\frac{8 \varepsilon^{2}}{\kappa^{2}-4 \varepsilon^{2}} .
$$

This is the variance of the photon number for the two signal light beams. This variance is four times the variance of the photon number obtained in Ref. [1]-[8].

On the other hand, substitution of (35) into Equation (44) results in 


$$
(\Delta n)_{\text {out }}^{2}=\kappa^{2}(\Delta n)^{2}
$$

in which

$$
(\Delta n)_{\text {out }}^{2}=\left\langle\hat{a}_{\text {out }}^{+2} \hat{a}_{\text {out }}^{2}\right\rangle+2 \kappa \bar{n}_{\text {out }}-\bar{n}_{\text {out }}^{2} .
$$

This shows that the variance of the photon number of the output light is $\kappa^{2}$ times that of the cavity light which holds for the normally-ordered operators.

Furthermore, the energy fluctuations for the cavity mode, is defined by

$$
(\Delta E)^{2}=\left\langle\hat{H}^{2}\right\rangle-\langle\hat{H}\rangle^{2},
$$

then, using the commutation relation

$$
\left[\hat{E}^{+}, \hat{E}\right]=2 \hbar \omega,
$$

and combination of (44) and (53) along with (38) and (39) results in

$$
(\Delta E)^{2}=\hbar^{2} \omega^{2}(\Delta n)^{2} .
$$

This shows that the energy fluctuation is equal to the product of the square of photon energy and the photon number fluctuation.

\section{Quadrature Squeezing}

We wish here to study the squeezing properties of the two signal light beams produced by one-mode subharmonic generator. We define the photon number quadrature variance for a pair of superposed cavity light beams by

$$
\left(\Delta a_{ \pm}\right)^{2}=2+\left\langle: \hat{a}_{ \pm}(t), \hat{a}_{ \pm}(t):\right\rangle
$$

where

$$
\hat{a}_{+}(t)=\hat{a}^{+}(t)+\hat{a}(t)
$$

and

$$
\hat{a}_{-}(t)=i\left(\hat{a}^{+}(t)-\hat{a}(t)\right),
$$

are the plus and minus photon number quadrature operators for the cavity light. The first term on the right hand side of Equation (56) represents the photon number quadrature variance for a pair of superposed coherent light beams. It is also the commutation relation for the photon number annihilation and creation operators representing the superposed light beams. Now employing the $Q$ function described by Equation (25), one can easily verify that

$$
\langle\hat{a}(t)\rangle=\left\langle\hat{a}^{+}(t)\right\rangle=0 .
$$

On account of (57), (58), and (59), Equation (56) can be put in the form

$$
\left(\Delta a_{ \pm}\right)^{2}=2+2\left\langle\hat{a}^{+} \hat{a}\right\rangle \pm\left\langle\hat{a}^{+2}\right\rangle \pm\left\langle\hat{a}^{2}\right\rangle .
$$

Then using Equation (25), the expectation value of $\hat{a}^{2}$ can be written as

$$
\left\langle\hat{a}^{2}\right\rangle=\left.\frac{1}{\pi^{2}}\left[\frac{l^{2}-m^{2}}{l^{2}-m^{2}-4 l+4}\right]^{1 / 2} \frac{\mathrm{d}^{2}}{\mathrm{~d} a^{2}} \int \mathrm{d}^{2} \alpha \mathrm{d}^{2} \beta \exp \left[-\alpha^{*} \alpha-\beta^{*} \beta+\alpha \beta^{*}+\beta \alpha^{*}+a \alpha-u \alpha^{*} \beta-v \beta^{2}-v \alpha^{* 2}\right]\right|_{a=0},
$$

so that upon carrying out the integration, applying the relation given by (24), and performing the differentiation 
with respect to a and introducing the condition $a=0$, we readily find

$$
\left\langle\hat{a}^{2}\right\rangle=-\frac{2 v^{2}}{u^{2}-4 v^{2}} \text {. }
$$

Hence in view of (26), (27) along with (2), (3), and (5), there follows

$$
\left\langle\hat{a}^{2}\right\rangle=-\frac{\varepsilon}{(\kappa+2 \varepsilon)}\left(1-\mathrm{e}^{-(\kappa+2 \varepsilon) t}\right)-\frac{\varepsilon}{(\kappa-2 \varepsilon)}\left(1-\mathrm{e}^{-(\kappa-2 \varepsilon) t}\right) .
$$

With the aid of (33), (60), and (63), we easily get

$$
\left(\Delta a_{ \pm}\right)^{2}=2 \mp \frac{4 \varepsilon}{(\kappa \pm 2 \varepsilon)}\left(1-\mathrm{e}^{-(\kappa \pm 2 \varepsilon) t}\right) .
$$

This is the photon number quadrature variance of the two signal light beams which is twice of the quadrature variance obtained in Ref. [1]-[8]. In addition we observe that the signal mode is in a squeezed state and the squeezing occurs in the plus quadrature. Moreover, applying (35) in Equation (60), we get

$$
\left(\Delta a_{ \pm}\right)_{\text {out }}^{2}=\kappa\left(\Delta a_{ \pm}\right)^{2},
$$

in which

$$
\left(\Delta a_{ \pm}\right)_{\text {out }}^{2}=2 \kappa+2\left\langle\hat{a}_{\text {out }}^{+} \hat{a}_{\text {out }}\right\rangle \pm\left\langle\hat{a}_{\text {out }}^{+2}\right\rangle \pm\left\langle\hat{a}_{\text {out }}^{2}\right\rangle .
$$

We observe that the photon number quadrature variance of the output light is $\kappa$ times that of the cavity light.

On the other hand, photon-energy quadrature variance for the cavity light is defined by

$$
\left(\Delta E_{ \pm}\right)^{2}=2 \hbar \omega+\left\langle: \hat{E}_{ \pm}(t), \hat{E}_{ \pm}(t):\right\rangle,
$$

where

$$
\hat{E}_{+}(t)=\hat{E}^{+}(t)+\hat{E}(t)
$$

and

$$
\hat{E}_{-}(t)=i\left(\hat{E}^{+}(t)-\hat{E}(t)\right),
$$

are the plus and minus photon-energy quadrature operators for the cavity light. The first term on the right hand side of Equation (67) represents the photon energy quadrature variance for a pair of superposed coherent light beams. It is also the commutation relation for the photon energy annihilation and creation operators representing the superposed light beams.

Then employing (54), (60), (64), (68), and (69) along with (38) and (39), we put Equation (67) in the form

$$
\left(\Delta E_{ \pm}\right)^{2}=2 \hbar \omega \mp \frac{4 \hbar \omega \varepsilon}{(\kappa \pm 4 \varepsilon)}\left(1-\mathrm{e}^{-(\kappa \pm 2 \varepsilon) t}\right),
$$

in which $2 \hbar \omega$ being photon-energy quadrature variance of a pair of superposed coherent light beams. This equation can be rewritten, at steady state, as

$$
\left(\Delta E_{ \pm}\right)^{2}=\hbar \omega\left[2 \mp \frac{4 \varepsilon}{(\kappa \pm 4 \varepsilon)}\right] .
$$

Hence by comparing Equations (64) and (71), we observe that the photon-energy quadrature variance is equal to the product of the photon energy and the photon number quadrature variance.

Next we seek to obtain the quadrature squeezing of the two signal light beams relative to the quadrature variance of a pair of superposed coherent light beams. We thus define the photon number quadrature squeezing of the cavity signal mode by [1] 


$$
S_{+}=\frac{2-\left(\Delta a_{+}\right)^{2}}{2}
$$

so that on account of (64), there follows

$$
S_{+}=\frac{2 \varepsilon}{(\kappa+2 \varepsilon)}\left(1-\mathrm{e}^{-(\kappa+2 \varepsilon) t}\right) .
$$

Moreover, at steady-state and at threshold, the photon number quadrature squeezing turns out to be

$$
S_{+}=\frac{1}{2} \text {. }
$$

We note that at steady state and at threshold there is a $50 \%$ squeezing of the signal mode below the cavity coherent-state level.

On the other hand, we define the photon number quadrature squeezing of the output signal mode by [1]

$$
S_{+}=\frac{2 \kappa-\left(\Delta a_{+}\right)_{o u t}^{2}}{2 \kappa}
$$

where $2 \kappa$ is the photon number quadrature variance of a pair of superposed output coherent light beams. Hence combination of (64), (65), and (75) there emerges

$$
S_{+}=S_{+}^{\text {out }} .
$$

This indicates that the photon number quadrature squeezing of the output signal mode is exactly equal to that of the cavity signal mode.

Furthermore, we can also define photon-energy quadrature squeezing the cavity signal mode by

$$
S_{+}=\frac{2 \hbar \omega-\left(\Delta E_{+}\right)^{2}}{2 \hbar \omega} .
$$

Thus in view of (70) and (77), we have

$$
S_{+E}=S_{+}=S_{+}^{\text {out }},
$$

which indicates that photon-energy quadrature squeezing is the same as that of the photon number quadrature squeezing.

\section{Conclusions}

In this paper, we studied photon statistics and quadrature squeezing of the two signal light beams produced by one-mode sub-harmonic generator. So far we know that the Hamiltonian describing the process of sub-harmonic generation consists of the operators' $a^{2}$ and $a^{+2}$. And the quantum analysis of the signal mode is usually carried out employing the operators $\hat{a}$ and $\hat{a}^{+}$. Such analysis leads to one-half of the mean photon number of the signal mode. This problem is resolved by superposing the $Q$ function of the individual set of photons (the signal mode), each set consisting of one photon from each pair. On account of this, we obtained the $Q$ function of the two signal light beams (the signal mode) produced by a one-mode sub-harmonic generator. Employing this $Q$ function, we determined the mean energy, the energy fluctuations, the photon energy quadrature fluctuations, and the photon energy quadrature squeezing.

It is found that the mean photon energy (the mean photon number) of the signal mode is twice of a twin signal light beam. And the energy fluctuations (the variance of the photon number) of the two signal light beams is four times that of one of the signal light beam. We have also shown that the photon energy (the photon number) quadrature squeezing of the output signal mode is exactly equal to that of the cavity signal mode. In light of this, the global quadrature squeezing of the signal mode is independent of the mean values. On the basis of the above results, we come to the conclusion that the quantum analysis of a one-mode sub-harmonic generation should somehow be modified. 


\section{References}

[1] Kassahun, F. (2012) The Quantum Analysis of Light. Create Space, South Carolina.

[2] Anwar, J. and Zubairy, M.S. (1992) Physical Review A, 45, 1804. http://dx.doi.org/10.1103/PhysRevA.45.1804

[3] Daniel, B. and Fesseha, K. (1998) Optics Communications, 151, 384. http://dx.doi.org/10.1016/S0030-4018(98)00039-X

[4] Milburn, G.J. and Walls, D.F. (1983) Physical Review A, A27, 392. http://dx.doi.org/10.1103/PhysRevA.27.392

[5] Kassahun, F. (1998) Optics Communications, 156, 145. http://dx.doi.org/10.1016/S0030-4018(98)00425-8

[6] Plimark, L.I. and Walls, D.F. (1994) Physical Review A, A50, 2627. http://dx.doi.org/10.1103/PhysRevA.50.2627

[7] Walls, D.F. and Milburn, G.J. (1995) Quantum Optics. Springer-Verlag, Berlin.

[8] Scully, M.O. and Zubairy, M.S. (1997) Quantum Optics. Cambridge University Press, Cambridge. http://dx.doi.org/10.1017/CBO9780511813993

[9] Zhan, Y. (2010) Modern Applied Science, 4, 8.

[10] Agarwal, G.S. and Adam, G. (1989) Physical Review A, A39, 6259. http://dx.doi.org/10.1103/PhysRevA.39.6259

[11] Collett, M.J. and Gardiner, C.W. (1984) Physical Review A, A30, 1386. http://dx.doi.org/10.1103/PhysRevA.30.1386

[12] Lugiato, L.A. and Stkini, G. (1982) Optics Communications, 41, 67. http://dx.doi.org/10.1016/0030-4018(82)90215-2

[13] Walls, D.F. and Barakat, R. (1970) Physical Review A, A1, 446. http://dx.doi.org/10.1103/PhysRevA.1.446

[14] Milburn, G.J. and Walls, D.F. (1981) Optics Communications, 39, 401. 
Scientific Research Publishing (SCIRP) is one of the largest Open Access journal publishers. It is currently publishing more than 200 open access, online, peer-reviewed journals covering a wide range of academic disciplines. SCIRP serves the worldwide academic communities and contributes to the progress and application of science with its publication.

Other selected journals from SCIRP are listed as below. Submit your manuscript to us via either submit@scirp.org or Online Submission Portal.
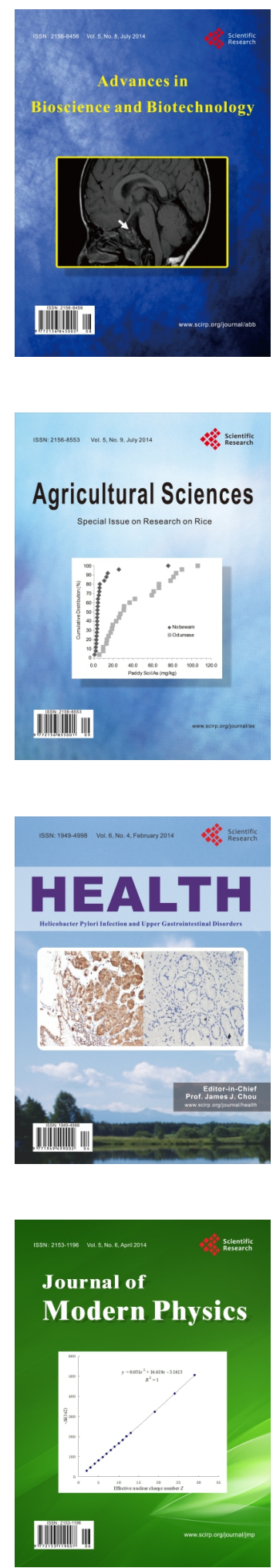
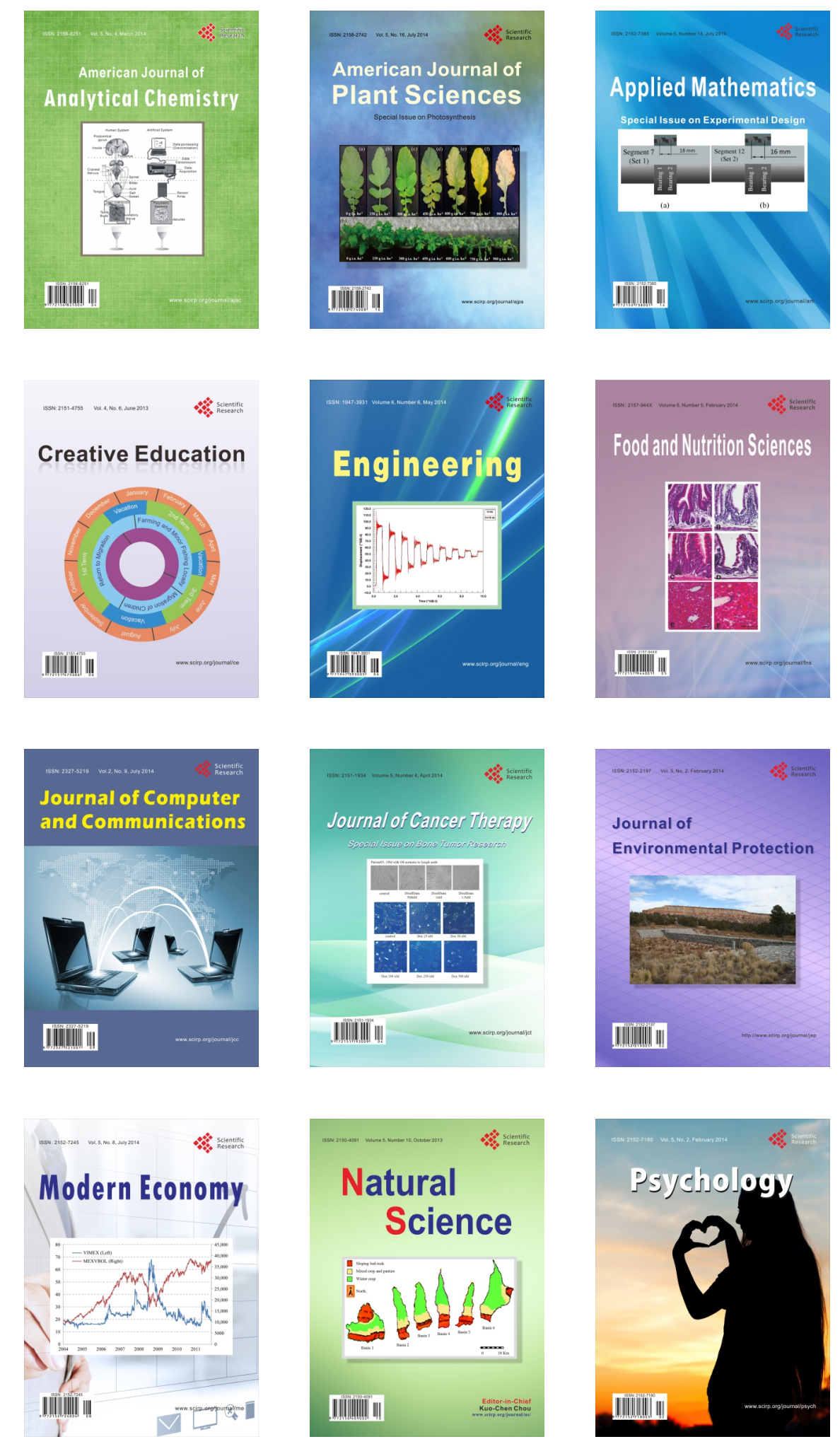